$1-1-1994$

\title{
Boundary and Interior Layer Behavior in a Time-dependent Singularly Perturbed System
}

S. Shao

Cleveland State University, s.shao@csuohio.edu

Follow this and additional works at: https://engagedscholarship.csuohio.edu/scimath_facpub

Part of the Mathematics Commons

How does access to this work benefit you? Let us know!

\section{Repository Citation}

Shao, S., "Boundary and Interior Layer Behavior in a Time-dependent Singularly Perturbed System" (1994). Mathematics Faculty Publications. 331.

https://engagedscholarship.csuohio.edu/scimath_facpub/331

This Article is brought to you for free and open access by the Mathematics and Statistics Department at EngagedScholarship@CSU. It has been accepted for inclusion in Mathematics Faculty Publications by an authorized administrator of EngagedScholarship@CSU. For more information, please contact library.es@csuohio.edu. 


\title{
BOUNDARY AND INTERIOR LAYER BEHAVIOR IN A TIME-DEPENDENT SINGULARLY PERTURBED SYSTEM
}

\author{
S. SHAO
}

Key words and phrases: Boundary layer, interior layer, singularly perturbed system.

\section{INTRODUCTION}

WE CONSIDER the time-dependent singularly perturbed system of partial differential equations

$$
\begin{aligned}
& u_{t}=u_{x x}-v \quad(x, t) \text { in }(0,1) \times(0, \infty) \text {, } \\
& u(x, 0, \varepsilon)=\phi_{1}(x, \varepsilon) \quad x \text { in }[0,1], \\
& u(0, t, \varepsilon)=u(1, t, \varepsilon)=0 \quad t \text { in }[0, \infty), \\
& v_{t}-f\left(u, u_{x}\right) v_{x}+g\left(x, u, u_{x}\right) v=\varepsilon v_{x x} \quad(x, t) \text { in }(0,1) \times(0, \infty), \\
& v(x, 0, \varepsilon)=\phi_{2}(x, \varepsilon) \quad x \text { in }[0,1], \\
& v(0, t, \varepsilon)=v_{0}, \quad v(1, t, \varepsilon)=v_{1} \quad t \text { in }[0, \infty),
\end{aligned}
$$

where $\varepsilon$ is a small positive parameter, $f\left(u, u_{x}\right)=h(u)$ or $h\left(u_{x}\right), h(0)=0$ and $g\left(x, u, u_{x}\right) \geq 0$. We assume that $\partial f / \partial u$ and $\partial f / \partial u^{\prime}$ do not change sign in $[0,1] \times[0, \infty), \phi_{i}(i=1,2)$ are continuous and $\left|\phi_{i}\right|_{\infty}(i=1,2)$ are bounded in $[0,1]$ for all $\varepsilon>0$, where $|\phi|_{\infty}$ is defined as the $L^{\infty}$-norm of $\phi$, finally $f\left(u, u_{x}\right)$ and $g\left(x, u, u_{x}\right)$ are of class $C^{(1)}[0,1]$. The corresponding steady-state equations of such a system have been studied by Dorr, Parter and Shampine [1, 2], Howes and Shao [3], Harris and Shao [4] and Shao [5]. The steady-state solution (time $t$ tends to infinity) of (1.1) is stable with respect to all initial perturbations as $\varepsilon \rightarrow 0^{+}$in $[0,1]$ and the solution $v$ is asymptotically stable except at the points which the function $f\left(u, u_{x}\right)$ vanishes (cf. [5]). It is natural to seek an answer to whether the steady-state solution can be generalized to the timedependent system (1.1). Moreover, such equations in (1.1) are related to a physical model of the homogenization of a passive tracer in a flow with closed mean streamlines. Rhines and Young [6] discussed the process of expulsion of the gradient of the passive scalar, they studied the initial-value problem

$$
\begin{gathered}
\theta_{t}+J(\psi, \theta)=k \nabla^{2} \theta, \\
\theta(x, y, 0)=\theta_{0}(x, y),
\end{gathered}
$$

where $\psi(x, y, t)$ is the streamfunction of the flow, $\theta$ is the passive scalar and $k$ is the diffusivity. In a steady plane shear-flow, the tracer equation becomes $\theta_{t}+U \theta_{x}=k \nabla^{2} \theta$, here $U$ is the velocity of the flow. For certain initial values of $\theta$ (for example, $\theta(x, y, 0)=\cos k x$ or $\sin k x$ ), Rhines and Young derived mathematical expressions of the solution of system (1.2) and gave a physical explanation of how rapidly the passive scalar $\theta$ is homogenized by interacting the 
velocity field and the diffusivity. However, for small $k$, the behavior of the solutions $\theta$ of the system with more general initial conditions is not known. The $v$-equation of (1.1) is a simple analogue of Rhines and Youngs' model with a more general initial condition $v(x, t, \varepsilon)=\phi_{2}(x, \varepsilon)$.

In the spirit of Howes [7], applying singular perturbation theory and utilizing differential inequality techniques, we first provide the asymptotic behavior of the solutions as $\varepsilon \rightarrow 0^{+}$of system (1.1) for a finite time $(t \leq T<\infty)$. The asymptotic solutions of (1.1) are in terms of the solutions of the associated reduced equations

$$
\begin{gathered}
U_{t}=U_{x x}-V, \quad U(x, 0)=\phi_{1}(x), \quad U(0, t)=U(1, t)=0, \\
V_{t}-f\left(U, U_{x}\right) V_{x}+g\left(x, U, U_{x}\right) V=0, \\
V(x, 0)=\phi_{2}(x), \quad V(0, t)=v_{0}, \quad V(1, t)=v_{1}
\end{gathered}
$$

and supplemented by appropriate boundary layers as well as interior layers. Our results show that the initial data of $u$ and $v$ play critical roles in the determination of the asymptotic solutions of system (1.1) as $\varepsilon \rightarrow 0^{+}$for the case $f\left(u, u_{x}\right)=h(u)$, the boundary data of $v$ will effect the case $f\left(u, u_{x}\right)=h\left(u_{x}\right)$ as the steady-state solution of the system. We hope our results may provide deeper understanding of the related physical problems, such as some problems in fluid dynamics.

\section{DEVELOPMENT}

Let $\varepsilon=0$, we consider the associated reduced system of partial differential equations

$$
\begin{gathered}
U_{t}=U_{x x}-V, \\
U(x, 0)=\phi_{1}(x), \quad U(0, t)=U(1, t)=0, \\
V_{t}-f\left(U, U_{x}\right) V_{x}+g\left(x, U, U_{x}\right) V=0, \\
V(x, 0)=\phi_{2}(x), \quad V(0, t)=v_{0}, \quad V(1, t)=v_{1} .
\end{gathered}
$$

We notice that the $V$-equation of (2.1) is a reaction-convection-diffusion equation. The $U$-equation (as well as the $u$-equation in (1.1)) of (2.1) is an initial-boundary value problem for the nonhomogeneous heat equation and its solutions are explicitly known (cf. [8, 9]). The exact solution $U(x, t)$ can be obtained by classical methods. That is,

$$
U(x, t)=\sum_{n=1}^{\infty} 2 \mathrm{c}^{-(n \pi)^{2} t}\left[\int_{0}^{1}\left\{\phi_{1}(x) \cdots \int_{0}^{1} V(x, \tau) \mathrm{e}^{(n \pi)^{2} \tau} \mathrm{d} \tau\right\} \sin n \pi x \mathrm{~d} x\right] \sin n \pi x .
$$

The solution $U=U(x, t)$ depends on the initial condition $\phi_{1}$ and the solution $V=V(x, t)$ of the $V$-equation of (2.1). For a finite time $t \leq T$, there exists a locally unique solution of (1.1) as $\varepsilon \rightarrow 0^{+}$which is close to a solution of (2.1), except in the neighborhoods of the boundary layer and interior layer where the second derivative of $v$ becomes large as $\varepsilon \rightarrow 0^{+}$(cf. [7]). We look for such a solution of (1.1) as $\varepsilon \rightarrow 0^{+}$by seeking a reduced solution $(U, V)$ of $(2.1)$, which satisfies the original data on the subsets of the parabolic boundary $B=\{(0, t): 0<t<T\} \cup$ $\{(x, 0): 0 \leq x \leq 1\} \cup\{(1, t): 0<t<T\}$ where the characteristic curves of the $V$-equation enter $\Omega=(0,1) \times(0, T)$. The solution $(U, V)$ supplemented by appropriate boundary layer and 
interior layer correction terms describes the corrected asymptotic behavior of the solutions of (1.1) as $\varepsilon \rightarrow 0^{+}$(cf. [7]).

We begin by introducing some results in [7] to study the $V$-equation of (2.1)

$$
V_{t}-f\left(U, U_{x}\right) V_{x}+g\left(x, U, U_{x}\right) V=0
$$

The characteristic direction of (VE) equals $\left[-f\left(U, U_{x}\right), 1\right]$. We define $\omega(x, t)=t x(x-1)$,

$$
\sigma(x, t)=\left[-f\left(U, U_{x}\right), 1\right] \cdot\left[\frac{\partial \omega}{\partial x}, \frac{\partial \omega}{\partial t}\right] \quad \text { and } \quad B=B_{<} \cup B_{0} \cup B_{>},
$$

here

$$
\begin{gathered}
B_{<}=\{(x, t) \in B: \sigma(x, t)<0\}, \\
B_{0}=\{(x, t) \in B: \sigma(x, t)=0\}, \\
B_{>}=\{(x, t) \in B: \sigma(x, t)>0\} .
\end{gathered}
$$

Then we have $\Omega=(0,1) \times(0, T)=\{(x, t): \omega(x, t)<0\}$ and $[\partial \omega / \partial x, \partial \omega / \partial t]$ is the exterior normal to the boundary $B \backslash\{(0,0)\} \backslash\{(1,0)\}$. Therefore, we seek the reduced solution $V$ of (VE) that must satisfy the given initial-boundary data on $B_{<}$. The boundary layer of width $O(\varepsilon)$ occurs on $B_{>}$and we have a boundary layer of width $O\left(\varepsilon^{1 / 2}\right)$ on $B_{0}$ because the boundary layer itself is a characteristic [7]. Moreover, (i) $V(0, t)=v_{0}$ if $f\left(U(0, t), U_{x}(0, t)\right)<0$ for all $t$ in [0,T]; (ii) $V(1, t)=v_{1}$ if $f\left(U(1, t), U_{x}(1, t)\right)>0$ for all $t$ in [0,T]; (iii) if $f\left(U(0, t), U_{x}(0, t)\right)$ or $f\left(U(1, t), U_{x}(1, t)\right)$ change sign in $[0, T]$, then $V(i, t)=v_{i}$ if $f^{*}\left(U(i, t), U_{x}(i, t)\right)<0$ for some $t$ in $[0, T], i=0,1$, here

$$
f^{*}(U(i, t), U x(i, t))= \begin{cases}f\left(U(0, t), U_{x}(0, t)\right) & \text { if } i=0 \\ -f\left(U(1, t), U_{x}(1, t)\right) & \text { if } i=1\end{cases}
$$

These results follow from $\sigma(0, t)<0$ if $f\left(U(0, t), U_{x}(0, t)\right)<0$ and $\sigma(1, t)<0$ if

$$
f\left(U(1, t), U_{x}(1, t)\right)>0
$$

for all $t$ in $[0, T]$. Since

$$
\begin{aligned}
\sigma(x, 0) & =\left[-f\left(U(x, 0), U_{x}(x, 0)\right), 1\right] \cdot\left[\frac{\partial \omega}{\partial x}, \frac{\partial \omega}{\partial t}\right]_{t=0} \\
& =\left(-f\left(U(x, 0), U_{x}(x, 0)\right), 1\right) \cdot(0,-1) \equiv-1<0,
\end{aligned}
$$

we have that the reduced solution $V$ satisfies $V(x, 0)=\phi_{2}(x)$. Therefore, it turns out that we need to determine the behavior of $f\left(U, U_{x}\right)$ to solve system (1.1). In order to simplify our following discussion, we study the two cases (A) $f\left(u, u_{x}\right)=h(u)$ and (B) $f\left(u, u_{x}\right)=h\left(u_{x}\right)$ separately. 
3. CASE A: $f\left(u, u_{x}\right)=h(u)$

Consider now the following initial-boundary value problem

$$
\begin{gathered}
u_{t}=u_{x x}-v, \quad(x, t) \text { in }(0,1) \times(0, T), \\
u(x, 0, \varepsilon)=\phi_{1}(x, \varepsilon), \quad u(0, t, \varepsilon)=u(1, t, \varepsilon)=0, \quad x \text { in }[0,1], t \text { in }[0, T], \\
v_{t}-h(u) v_{x}+g\left(x, u, u_{x}\right) v=\varepsilon v_{x x}, \quad(x, t) \text { in }(0,1) \times(0, T), \\
v(x, 0, \varepsilon)=\phi_{2}(x, \varepsilon), \quad x \text { in }[0,1], \\
v(0, t, \varepsilon)=v_{0}, \quad v(1, t, \varepsilon)=v_{1}, \quad t \text { in }[0, T] .
\end{gathered}
$$

The reduced equations of (MI)

$$
\begin{gathered}
U_{t}=U_{x x}-V, \\
U(x, 0)=\phi_{1}(x), \quad U(0, t)=U(1, t)=0, \\
V_{t}-h(U) V_{x}+g\left(x, U, U_{x}\right) V=0, \\
V(x, 0)=\phi_{2}(x), \quad V(0, t)=v_{0}, \quad V(1, t)=v_{1} .
\end{gathered}
$$

Since $h(U(0, t))=0=h(U(1, t))$ by $h(0)=0$ for all $t$ in $[0, T]$, we have $x=0$ and $x=1$ are also characteristic curves of the $V$-equation. These dramatically simplify our studies of the solutions of (R1) because the characteristic curves of the $V$-equation enter $\bar{\Omega}=[0,1] \times[0, T]$ through $t=0$ only; see Fig. 1 (note that the characteristics are not straight lines unless the function $h(U(x, t))$ is a constant). It follows that the initial value problem of the $V$-equation has the solution which satisfies

$$
V(x, t)=\phi_{2}\left(x-\int_{0}^{t} h(U(x(\tau), \tau)) \mathrm{d} \tau\right)\left[\exp \left\{-\int_{0}^{t} g\left(x, U(x, \tau), U_{x}(x, \tau)\right) \mathrm{d} \tau\right\}\right]
$$

on $B_{<}=\{(x, t) \in B: \sigma(x, t)<0\}=B$. The boundary layer of width $O\left(\varepsilon^{1 / 2}\right)$ displays at $x=0$ and $x=1$ (cf. Section 2). Therefore, as $\varepsilon \rightarrow 0^{+}$we have the solution of (MI)

$$
\begin{aligned}
v(x, t, \varepsilon)= & \phi_{2}\left(x-\int_{0}^{t} h(U(x(\tau), \tau)) \mathrm{d} \tau\right)\left[\exp \left\{-\int_{0}^{t} g\left(x, U(x, \tau), U_{x}(x, \tau)\right) \mathrm{d} \tau\right\}\right] \\
& +B L(x=0)+B L(x=1)
\end{aligned}
$$

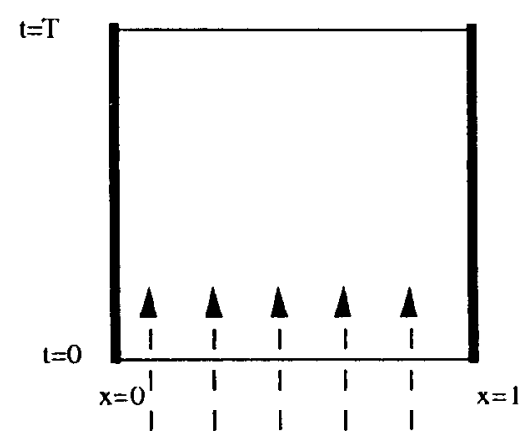

Fig. 1. The characteristics of the $V$-equation. 
and

$$
\begin{aligned}
& u(x, t, \varepsilon)=\sum_{n=1}^{\infty} 2 \mathrm{e}^{-(n \pi)^{2} t}\left[\int_{0}^{1}\left\{\phi_{1}(x)-\int_{0}^{t} v(x, \tau, \varepsilon) \mathrm{e}^{(n \pi)^{2} \tau} \mathrm{d} \tau\right\} \sin (n \pi x) \mathrm{d} x\right] \sin (n \pi x) \\
& \text { for }(x, t) \text { in } \bar{\Omega} \text {, }
\end{aligned}
$$

here if $g=0$

$$
B L(x=0)=O\left(v_{0} \operatorname{erf}\left[\frac{x \exp [-a t]}{\varepsilon^{1 / 2} \sqrt{2 a \exp (2 a t-1)}}\right]\right)
$$

and

$$
B L(x=1)=O\left(v_{1} \operatorname{erf}\left[\frac{(1-x) \exp [-b t]}{\varepsilon^{1 / 2} \sqrt{2 b \exp (2 b t-1)}}\right]\right)
$$

$a$ and $b$ are constants, erf is the error function.

We note that the structures of the boundary layers in (3.2) are invariant under different boundary values $v$. We shall make a comparison between (3.2) and the steady-state solution of system (MI) in Section 5 .

\section{CASE B: $f\left(u, u_{x}\right)=h\left(u_{x}\right)$}

We first consider the second submodel of $(1.1)$ where $f\left(u, u_{x}\right)=h\left(u_{x}\right), g \equiv 0$,

$$
\begin{gathered}
u_{t}=u_{x x}-v, \quad(x, t) \text { in }(0,1) \times(0, T), \\
u(x, 0, \varepsilon)=\phi_{1}(x, \varepsilon), \quad u(0, t, \varepsilon)=u(1, t, \varepsilon)=0, \quad x \text { in }[0,1], t \text { in }[0, T] \\
v_{t}+h\left(u_{x}\right) v_{x}=\varepsilon v_{x x}, \quad(x, t) \text { in }(0,1) \times(0, T), \\
v(x, 0, \varepsilon)=\phi_{2}(x, \varepsilon), \quad x \text { in }[0,1], \\
v(0, t, \varepsilon)=v_{0}, \quad v(1, t, \varepsilon)=v_{1}, \quad t \text { in }[0, T]
\end{gathered}
$$

and its associated reduced system of partial differential equations

$$
\begin{gathered}
U_{t}=U_{x x}-V, \\
U(x, 0)=\phi_{1}(x), \quad U(0, t)=U(1, t)=0, \\
V_{t}-h\left(U_{x}\right) V_{x}=0, \\
V(x, 0)=\phi_{2}(x), \quad V(0, t)=v_{0}, \quad V(1, t)=v_{1} .
\end{gathered}
$$

We see immediately that $U_{x} \neq 0$ at both $x=0$ and $x=1$ even though $U(0, t)=U(1, t)=0$. It makes it possible for the characteristic curves of the $V$-equation to enter $\bar{\Omega}$ through the boundary $x=0$ or $x=1$. To illustrate the effect of the driving term $h\left(U_{x}\right)$ on the behavior of solutions of the $V$-equation, we consider first the solution $U$ of (R2),

$$
U=U(x, t)=\sum_{n=1}^{\infty} 2 \mathrm{e}^{-(n \pi)^{2} t}\left[\int_{0}^{1}\left\{\phi_{1}(x)-\int_{0}^{t} V(x, \tau) \mathrm{e}^{(n \pi)^{2} \tau} \mathrm{d} \tau\right\} \sin n \pi x \mathrm{~d} x\right] \sin (n \pi x) .
$$

The partial derivative of $U$ with respect to $x$ is

$$
U_{x}(x, t)=\sum_{n=1}^{\infty} \frac{2 \mathrm{e}^{-(n \pi)^{2} t}}{n \pi}\left[\int_{0}^{1}\left\{\phi_{1}(x)-\int_{0}^{t} V(x, \tau) \mathrm{e}^{(n \pi)^{2} \tau} \mathrm{d} \tau\right\} \sin n \pi x \mathrm{~d} x\right] \cos (n \pi x)
$$


(we can differentiate this Fourier series $U$ term by term because $U(0, t)=U(1, t)=0$ and $U_{x}$ are piecewise smooth). Then we have the following theorem.

THEOREM. If $u(x, t, \varepsilon)$ and $v(x, t, \varepsilon)$ are solutions of (MII), then as $\varepsilon \rightarrow 0^{+}$,

$$
u(x, t, \varepsilon)=\sum_{n=1}^{\infty} 2 \mathrm{e}^{-(n \pi)^{2} t}\left[\int_{0}^{1}\left\{\phi_{1}(x)-\int_{0}^{t} v(x, \tau, \varepsilon) \mathrm{e}^{(n \pi)^{2} \tau} \mathrm{d} \tau\right\} \sin (n \pi x) \mathrm{d} x\right] \sin (n \pi x) ;
$$

(a) if $\int_{0}^{1} \phi_{1}(x) \sin (n \pi x) \mathrm{d} x \geq 0$ for each positive integer $n$, then

$$
\text { (i) } v(x, t, \varepsilon)=V+I L(x=t)+B L(x=1)+B L(x=0) \quad \text { for } v_{0} \leq 0, v_{1} \leq 0 \text {, }
$$

in addition, if $2 \int_{0}^{1} \phi_{1}(x) \sin (n \pi x) \mathrm{d} x+\left(v_{m}\left(1-\mathrm{e}^{-(n \pi)^{2} t}\right)\right) /(n \pi)^{3}<0$ for some $t \geq t_{i}(i=0,1)$, here $v_{m}=\max \left\{v_{0}, v_{1}\right\}$, then

$$
\text { (ii) } v(x, t, \varepsilon)=\left\{\begin{array}{l}
v_{0} \text { in } \Omega \mathrm{a} \\
V \text { in } \Omega \mathrm{b}
\end{array}+I L(L 1)+B L(x=1)+B L\left(x=0, t \leq t_{0}\right)\right.
$$

for $v_{0} \geq 0, v_{1} \leq 0$;

$$
\text { (iii) } v(x, t, \varepsilon)=\left\{\begin{array}{l}
v_{1} \text { in } \Omega \mathrm{c} \\
V \text { in } \Omega \mathrm{d}
\end{array}+I L(L 2)+B L(x=0)+B L\left(x=1, t \leq t_{1}\right)\right.
$$

for $v_{0} \leq 0, v_{1} \geq 0$

(iv) $v(x, t, \varepsilon)$

$$
=\left\{\begin{array}{l}
V^{*} \text { in } \Omega 2 \\
V \text { in } \Omega 1
\end{array}+I L\left(L 4, x \leq x^{*}\right)+I L\left(x=x^{*}, t \geq t^{*}\right)+I L\left(L 5, x \geq x^{*}\right)\right.
$$

for $v_{0} \geq 0, v_{1} \geq 0$, where

$$
V^{*}=\left\{\begin{array}{l}
v_{0} \text { in } \Omega 2 \mathrm{a}, \\
v_{1} \text { in } \Omega 2 \mathrm{~b}
\end{array} \quad \Omega 2=\Omega 2 \mathrm{a} \cup \Omega 2 \mathrm{~b}, \quad \Omega 1=\bar{\Omega} \backslash \Omega 2,\right.
$$

for the locations of $\Omega 2 \mathrm{a}, \Omega 2 \mathrm{~b}, L 3, L 4, L 5, t^{*}$ and $x^{*}$, see Fig. 4 ; $\Omega \mathrm{a}$ and $\Omega \mathrm{b}, \Omega \mathrm{c}$ and $\Omega \mathrm{d}$ are the subdomains in Figs 2 and 3, respectively, $L 1$ and $L 2$ are the characteristic curves of $V$-equations, see Figs 2 and 3.

(b) If $\int_{0}^{1} \phi_{1}(x) \sin (n \pi x) \mathrm{d} x \leq 0$ for each positive integer $n$, then

$$
\text { (i) } v(x, t, \varepsilon)=\left\{\begin{array}{l}
V^{* *} \text { in } \Omega 4 \\
V \text { in } \Omega 3
\end{array}+I L\left(L 4, x \leq x_{0}\right)+I L\left(x=x_{0}, t \geq t_{0}\right)+I L\left(L 5, x \geq x_{0}\right)\right.
$$

for $v_{0} \geq 0, v_{1} \geq 0$, where

$$
V^{* *}=\left\{\begin{array}{l}
v_{0} \text { in } \Omega 4 \mathrm{a}, \\
v_{1} \text { in } \Omega 4 \mathrm{~b}
\end{array} \quad \Omega 4=\Omega 4 \mathrm{a} \cup \Omega 4 \mathrm{~b}, \quad \Omega 3=\bar{\Omega} \backslash \Omega 4,\right.
$$

$\Omega 3, \Omega 4 \mathrm{a}$ and $\Omega 4 \mathrm{~b}$ are defined as the subdomains $\Omega 1, \Omega 2 \mathrm{a}$ and $\Omega 2 \mathrm{~b}$ in Fig. 4 . 


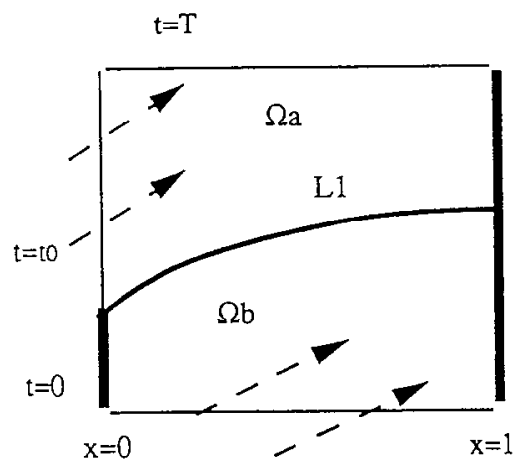

Fig. 2. The characteristics of the $V$-equation.

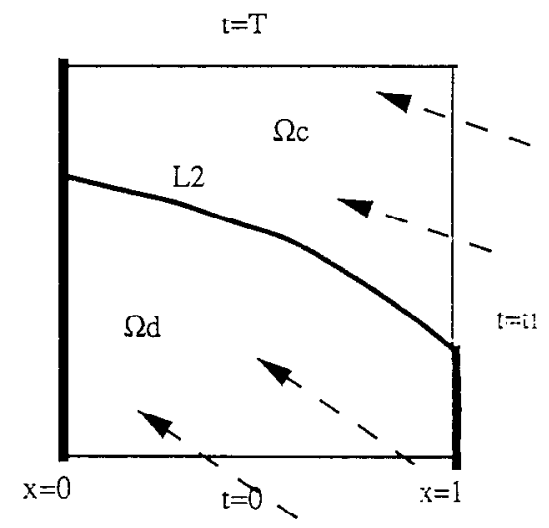

Fig. 3. The characteristics of the $V$-equation.

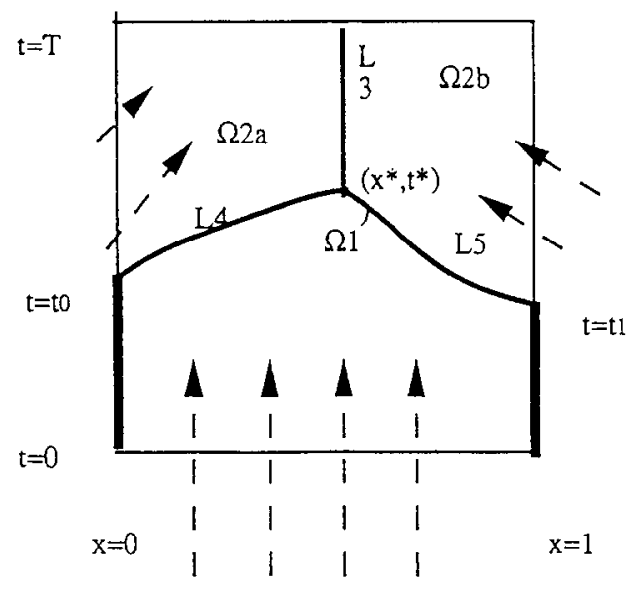

Fig. 4. The characteristics of the $V$-equation. 
(ii) $v(x, t, \varepsilon)=\left\{\begin{array}{l}v_{1} \text { in } \Omega \mathrm{c} \\ V \text { in } \Omega \mathrm{d}\end{array}+I L(L 2)+S L(x=0) \quad\right.$ for $v_{0} \leq 0, v_{1} \geq 0 ;$

(iii) $v(x, t, \varepsilon)=\left\{\begin{array}{l}v_{0} \text { in } \Omega \mathrm{a} \\ V \text { in } \Omega \mathrm{b}\end{array}+I L(L 1)+Z L(x=1) \quad\right.$ for $v_{0} \geq 0, v_{1} \leq 0 ;$

where $V(x, t)=\phi_{2}\left(x-\int_{0}^{t} h\left(U_{x}(x, \tau)\right) \mathrm{d} \tau\right), B L(x=i)$ represents the boundary layer at $x=i$ $(i=1,2)$ defined as $B L(x=i)=O\left(V(i, t)+\left(v_{i}-V(i, t)\right) \operatorname{cxp}\left[h(U x(i, t))(i-x) / \varepsilon^{1 / 2}\right]\right)$; while $I L(t+a x=b)$ represents an interior layer of a solution with the form

$$
\begin{aligned}
& W(x, t, \varepsilon)=\left\{\begin{array}{l}
w_{0} \text { for } t+a x \geq b \\
w_{1} \text { for } t+a x \leq b
\end{array}\right. \text { and } \\
& I L(t+a x=b)=O\left(\varepsilon^{1 / 2}\left|\frac{\partial w_{1}}{\partial n}-\frac{\partial w_{0}}{\partial n}\right| \exp \left[\frac{-|t+a x-b|}{\varepsilon^{1 / 2}}\right]\right) \\
& +O\left(\left|w_{1}-w_{0}\right| \exp \left[\frac{-(t+a x-b)^{2}}{\varepsilon^{1 / 2}}\right]\right)+O\left(\varepsilon^{1 / 2}\right),
\end{aligned}
$$

$a$ and $b$ are constants, $\partial w / \partial n$ defined by $[\partial \omega / \partial x, \partial \omega / \partial t] \cdot\left[n_{1}, n_{2}\right], n=\left[n_{1}, n_{2}\right]$ is the outer unit normal to the subdomain $\{(x, t): t+a x-b<0\} . S L(x=0)$ in (b) (ii) means an interior layer near $x=0$ with

$$
S L(x=0)=\left\{\begin{array}{l}
v_{0} \text { for } 0 \leq x \leq \delta \\
v_{1} \text { for } \delta \leq x \leq 1-\delta
\end{array}+O\left(\left(v_{1}-v_{0}\right) \exp \left[\frac{-(x-\delta / 2)^{2}}{\varepsilon^{1 / 2}}\right]\right)+O\left(\varepsilon^{1 / 2}\right),\right.
$$

and in (b) (iii),

$$
Z L(x=1)=\left\{\begin{array}{l}
v_{0} \text { for } 0 \leq x \leq 1-\delta \\
v_{1} \text { for } 1-\delta \leq x \leq 1
\end{array}+O\left(\left(v_{0}-v_{1}\right) \exp \left[\frac{-(x-1-\delta / 2)^{2}}{\varepsilon^{1 / 2}}\right]\right)+O\left(\varepsilon^{1 / 2}\right)\right.
$$

is an interior layer near $x=1$, and $0<\delta=O\left(\varepsilon^{1 / 2}\right)$.

Proof. Equation (4.3) follows immediately from (4.1). The initial value problem for the $V$ equation $V_{t}-h\left(U_{x}\right) V_{x}=0, V(x, 0)=\phi_{2}(x)$ has the solution $V(x, t)=\phi_{2}\left(x-\int_{0}^{t} h\left(U_{x}(x, \tau)\right) \mathrm{d} \tau\right)$ in some subset of $\Omega$. From (4.1), we have

and

$$
U_{x}(0, t)=\sum_{n=1}^{\infty} \frac{2 \mathrm{e}^{-(n \pi)^{2} t}}{n \pi}\left[\int_{0}^{1}\left\{\phi_{1}(x)-\int_{0}^{t} V(x, \tau) \mathrm{e}^{(n \pi)^{2} \tau} \mathrm{d} \tau\right\} \sin n \pi x \mathrm{~d} x\right]
$$

$$
U_{x}(1, t)=\sum_{n=1}^{\infty} \frac{2 \mathrm{e}^{-(n \pi)^{2} t}}{n \pi}\left[\int_{0}^{1}\left\{\phi_{1}(x)-\int_{0}^{t} V(x, \tau) \mathrm{e}^{(n \pi)^{2} \tau} \mathrm{d} \tau\right\} \sin n \pi x \mathrm{~d} x\right](-1)^{n} .
$$

Since $U_{x}(0, t)<0$ implies $h\left(U_{x}(0, t)\right)<0 \quad\left(U_{x}(1, t)\right)>0$ implies $h\left(U_{x}(1, t)\right)>0$ (cf. assumptions in Section 1), by the discussion in Section 2, the characteristic curves of the 
$V$-equation also enter $\bar{\Omega}$ along the boundary $x-0(x=1)$ if $U_{x}(0, t)<0\left(U_{x}(1, t)>0\right)$ for the portions of $t \geq x$. It also follows that

(C1) $V(x, t)=v_{0}$ if $U_{x}(0, t)<0$ for all $t \geq t_{0} \geq x$,

(C2) $V(x, t)=v_{1}$ if $U_{x}(1, t)>0$ for all $t \geq t_{0} \geq x$ (cf. Section 2).

In other words, if $U_{x}(0, t)>0$ for all $t \geq t_{0} \geq x$ then there must be a boundary layer at $x=0$, and $U_{x}(1, t)<0$ for all $t \geq t_{0} \geq x$ there is a likewise boundary layer at $x=1$. If both conditions (C1) and (C2) are satisfied then there exists an interior layer for some $t_{0} \geq x$ for $v_{0} \neq v_{1}$. Based on the above analysis and conditions, we can now verify our results.

(a) If $\int_{0}^{1} \phi_{1}(x) \sin (n \pi x) \mathrm{d} x \geq 0$ for each positive integer $n$, since

$$
U_{x}(0, t)=\sum_{n=1}^{\infty} \frac{2 \mathrm{e}^{-(n \pi)^{2} t}}{n \pi}\left[\int_{0}^{1} \phi_{1}(x) \sin (n \pi x) \mathrm{d} x+\frac{v_{0}\left(1-\mathrm{e}^{-(n \pi)^{2} t}\right)}{(n \pi)^{3}}\left(1-(-1)^{-n}\right)\right]>0
$$

and

$$
U_{x}(1, t)=\sum_{n=1}^{\infty} \frac{2 \mathrm{e}^{-(n \pi)^{2} t}}{n \pi}\left[\int_{0}^{1} \phi_{1}(x) \sin n \pi x \mathrm{~d} x+\frac{v_{1}\left(1-\mathrm{e}^{-(n \pi)^{2} t}\right)}{(n \pi)^{3}}\left(1-(-1)^{-n}\right)\right](-1)^{n}<0
$$

for $v_{0} \leq 0, v_{1} \leq 0$ and for all $t$ in $[0, T],(\mathrm{C} 1)$ and $(\mathrm{C} 2)$ imply that the only asymptotic behavior of the reduced solution of $V$ is then the boundary layer of width $O(\varepsilon)$ at both $x=0$ and $x=1$, and the characteristic curves of the $V$-equation enter $\bar{\Omega}$ through the boundary $t=0$ only. Therefore, the solution $v$ of (MII)

$$
v(x, t, \varepsilon)=V+I L(x=t)+B L(x=1)+B L(x=0) \quad \text { for } v_{0} \leq 0, v_{1} \leq 0
$$

as $\varepsilon \rightarrow 0^{+}$, where boundary layers $B L(x=i)(i=1,2)$ are defined by

$$
B L(x=i)=O\left(V(i, t)+\left(v_{i}-V(i, t)\right) \exp \left[h(U x(i, t))(i-x) / \varepsilon^{1 / 2}\right]\right),
$$

all the interior layers $I L$ will be provided at the end of our proof.

However, if $v_{0} \geq 0$ or $v_{1} \leq 0$ and also $2 \int_{0}^{1} \phi_{1}(x) \sin (n \pi x) \mathrm{d} x+\left(v_{0}\left(1-\mathrm{e}^{-(n \pi)^{2} t}\right)\right) /(n \pi)^{3}<0$ for some $t \geq t_{0}>x,(i=0,1)$, then

$$
U_{x}(0, t)=\sum_{n=1}^{\infty} \frac{2 \mathrm{e}^{-(n \pi)^{2} t}}{n \pi}\left[\int_{0}^{1} \phi_{1}(x) \sin (n \pi x) \mathrm{d} x+\frac{v_{0}\left(1-\mathrm{e}^{-(n \pi)^{2} t}\right)}{(n \pi)^{3}}\left(1-(-1)^{-n}\right)\right]<0
$$

for some $t \geq t_{0} \geq x$. The characteristic curves enter $\bar{\Omega}$ through the sides $t=0$ and the portion of $x=0$ with $t \geq t_{0}$; see Fig. 2. We then have $V(x, t)=v_{0}$ in $\Omega a$ by (C1) and (C2). The boundary displays at $x=1$ and $x=0$ with $t \leq t_{0}$. Since the reduced solution $V$ is nonsmooth at $L 1$ we must supplement it with an interior layer (cf. [7, 10]). The following results hold

$$
v(x, t, \varepsilon)=\left\{\begin{array}{l}
v_{0} \text { in } \Omega \mathrm{a} \\
V \text { in } \Omega \mathrm{b}
\end{array}+I L(L 1)+B L(x=1)+B L\left(x=0, t \leq t_{0}\right)\right.
$$

for $v_{0} \geq 0, v_{1} \leq 0, I L(L 1)$ represents interior layer at $L 1$.

For $v_{1} \geq 0, v_{0} \leq 0$, we have

$$
U_{x}(1, t)=\sum_{n=1}^{\infty} \frac{2 \mathrm{e}^{-(n \pi)^{2} t}}{n \pi}\left[\int_{0}^{1} \phi_{1}(x) \sin n \pi x \mathrm{~d} x+\frac{v_{1}\left(1-\mathrm{e}^{-(n \pi)^{2} t}\right)}{(n \pi)^{3}}\left(1-(-1)^{-n}\right)\right](-1)^{n}>0
$$


for some $t \geq t_{1} \geq x$ and $2 \int_{0}^{1} \phi_{1}(x) \sin (n \pi x) \mathrm{d} x+\left(v_{1}\left(1-\mathrm{e}^{-(n \pi)^{2} t}\right)\right) /(n \pi)^{3}<0$ for some $t \geq t_{i}>x$, it follows that $V(x, t)=v_{1}$ in $\Omega \mathrm{c}$ by (C1) and (C2). The characteristics $L 2$ of the $V$-equation in this case are illustrated in Fig. 3. By the same arguments as in case (ii), we obtain (iii)

$$
v(x, t, \varepsilon)=\left\{\begin{array}{l}
v_{1} \text { in } \Omega \mathrm{c} \\
V \text { in } \Omega \mathrm{d}
\end{array}+I L(L 2)+B L(x=0)+B L\left(x=1, t \leq t_{1}\right) \text { for } v_{0} \leq 0, v_{1} \geq 0 .\right.
$$

(iv) If $2 \int_{0}^{1} \phi_{1}(x) \sin (n \pi x) \mathrm{d} x+\left(v_{m}\left(1-\mathrm{e}^{-(n \pi)^{2} t}\right)\right)(n \pi)^{3}<0$ for some $t \geq t_{i}(i=0,1)$, for all $n$, and $v_{0} \geq 0, v_{1} \geq 0$, we have $U_{x}(0, t)<0$ and $U_{x}(1, t)>0$ for some $t \geq t_{i}(i=0,1)$, respectively. The reduced solution $V$ must satisfy both boundary conditions, that is, $V(0, t)=v_{0}$ for $t \geq t_{0}$ and $V(1, t)=v_{1}$ for $t \geq t_{1}$. It implies that there exists an interior layer at $\left(x^{*}, t^{*}\right)$ where $\left(x^{*}, t^{*}\right)$ is the intersection of three characteristics $L 4, L 5$ and $L 3$; see Fig. 4 . Another two interior layers display at $L 4$ for $x \leq x^{*}$ and $L 5$ for $x \geq x^{*}$ because of the nonsmoothness of $V$ along those two curves, $L 3: x=x^{*}, t>t^{*}$. Hence, as $\varepsilon \rightarrow 0^{+}$, the solution

$$
v(x, t, \varepsilon)=\left\{\begin{array}{l}
V^{*} \text { in } \Omega 2 \\
V \text { in } \Omega 1
\end{array}+I L\left(L 4, x \leq x^{*}\right)+I L\left(x=x^{*}, t \geq t^{*}\right)+I L\left(L 5, x \geq x^{*}\right)\right.
$$

for $v_{0} \geq 0, v_{1} \geq 0$ follows, here

$$
V^{*}=\left\{\begin{array}{l}
v_{0} \text { in } \Omega 2 \mathrm{a}, \\
v_{1} \text { in } \Omega 2 \mathrm{~b}
\end{array} \quad \Omega 2=\Omega 2 \mathrm{a} \cup \Omega 2 \mathrm{~b}, \quad \Omega 1=\bar{\Omega} \backslash \Omega 2,\right.
$$

for subdomains $\Omega 2 \mathrm{a}, \Omega 2 \mathrm{~b}$ and $\Omega 1$, see Fig. 4 .

The proof of all four cases in (a) is now complete.

(b) If $\int_{0}^{1} \phi_{1}(x) \sin (n \pi x) \mathrm{d} x \leq 0$ for each positive integer $n$, then $v_{0} \geq 0$ and $v_{1} \geq 0$ imply $U_{x}(0, t)<0$ and $U_{x}(1, t)>0$ for all $t$ in $[0,1]$ (cf. (4.4)). Conditions (C1) and (C2) tell us that $V(0, t)=v_{0}$ and $V(1, t)=v_{1}$ for all $t$ in $[0, T]$. Therefore, an inferior layer displays at $x=x_{0}$ in $(0,1)$ with $t \geq x_{0}$. Since

$$
V(x, t)=\phi_{2}\left(x-\int_{0}^{t} h\left(U_{x}(x(\tau), \tau)\right) \mathrm{d} \tau\right) \text { on } \Omega 3
$$

we must have the interior layer at $L 4$ with $x \leq x_{0}$ and $L 5$ with $x \geq x_{0}$ to supplement the reduced solution $V$ in this case. This then leaves us with the solution $v$ of (MII) satisfying (i)

$$
v(x, t, \varepsilon)=\left\{\begin{array}{l}
V^{* *} \text { in } \Omega 4 \\
V \text { in } \Omega 3
\end{array}+I L\left(L 4, x \leq x_{0}\right)+I L\left(x=x_{0}, t \geq t_{0}\right)+I L\left(L 5, x \geq x_{0}\right)\right.
$$

for $v_{0} \geq 0, v_{1} \geq 0$ as $\varepsilon \rightarrow 0^{+}$, where

$$
V^{* *}=\left\{\begin{array}{l}
v_{0} \text { in } \Omega 4 \mathrm{a}, \\
v_{1} \text { in } \Omega 4 \mathrm{~b}
\end{array} \quad \Omega 4=\Omega 4 \mathrm{a} \cup \Omega 4 \mathrm{~b},\right.
$$

$\Omega 3, \Omega 4 \mathrm{a}$ and $\Omega 4 \mathrm{~b}$ are defined as the subdomains $\Omega 1, \Omega 2 \mathrm{a}$ and $\Omega 2 \mathrm{~b}$ in Fig. 4. 
When $v_{0} \leq 0, v_{1} \geq 0$, we first notice that the reduced solution $V(0, t)=v_{1}$ for all $t$ in $[0, T]$, since $v_{1} \geq 0$ and $\int_{0}^{1} \phi_{1}(x) \sin (n \pi x) \mathrm{d} x \leq 0$ for each positive integer $n$ imply $U_{x}(1, t)>0$. Unfortunately,

$$
U_{x}(0, t)=\sum_{n=1}^{\infty} \frac{2 \mathrm{e}^{-(n \pi)^{2} t}}{n \pi}\left[\int_{0}^{1} \phi_{1}(x) \sin (n \pi x) \mathrm{d} x+\frac{v_{1}\left(1-\mathrm{e}^{-(n \pi)^{2} t}\right)}{(n \pi)^{3}}\left(1-(-1)^{-n}\right)\right]<0
$$

for all $t$ in $[0, T],(\mathrm{C} 1)$ tell us that the reduced solution $V$ must satisfy the boundary condition at $x=0$. However, since $v_{0} \leq 0$ in this case, the inequality of (4.5) is not true (in general), we have $V(0, t)=v_{0}$ on $x \leq \delta \ll 1$. The behavior of the reduced solution $V$ near $x=0$ is described by an interior layer called the $S$-layer $(S L(x=0))$ since $v_{0}<v_{1}$ with

$$
S L(x=0)=\left\{\begin{array}{r}
\begin{array}{l}
v_{0} \text { for } 0 \leq x \leq \delta \\
\end{array} \quad+\left(\left(v_{1}-v_{0}\right) \exp \left[\frac{-(x-\delta / 2)^{2}}{\varepsilon^{1 / 2}}\right]\right)+O\left(\varepsilon^{1 / 2}\right), \\
v_{1} \text { for } \delta \leq x \leq 1-\delta
\end{array}\right.
$$

and $V$ is also supported by an interior layer at $L 2$. The $v$ solution of (MII) satisfies (ii)

$$
v(x, t, \varepsilon)=\left\{\begin{array}{l}
v_{1} \text { in } \Omega \mathrm{c} \\
V \text { in } \Omega \mathrm{d}
\end{array}+I L(L 2)+S L(x=0) \text { for } v_{0} \leq 0, v_{1} \geq 0 \text { as } \varepsilon \rightarrow 0^{+} .\right.
$$

We finally reach the last case $v_{0} \geq 0, v_{1} \leq 0$ of (b). This case is just the reflection of the previous one. Let $y=1-x, M(y, t)=-U(1-y, t)$ and $N(y, t)=-V(1-y, t)$, then the previous results can be applied to the new system. Therefore, we obtain that the solution $v$ of the original system (MII) satisfies

$$
v(x, t, \varepsilon)=\left\{\begin{array}{l}
v_{0} \text { in } \Omega \mathrm{a} \\
V \text { in } \Omega \mathrm{b}
\end{array}+I L(L 1)+Z L(x=0) \text { for } v_{0} \geq 0, v_{1} \leq 0 \quad\right. \text { and }
$$

$\int_{0}^{1} \phi_{1}(x) \sin (n \pi x) \mathrm{d} x \leq 0$ for each $n$ as $\varepsilon \rightarrow 0^{+}$, where $Z L(x=1)$ is an interior layer near $x=1$ with

$$
Z L(x=1)=\left\{\begin{array}{l}
v_{0} \text { for } 0 \leq x \leq 1-\delta \\
v_{1} \text { for } 1-\delta \leq x \leq 1
\end{array}+O\left(\left(v_{0}-v_{1}\right) \exp \left[\frac{-(x-1-\delta / 2)^{2}}{\varepsilon^{1 / 2}}\right]\right)+O\left(\varepsilon^{1 / 2}\right)\right.
$$

and $0<\delta=O\left(\varepsilon^{1 / 2}\right)$.

Before we finish the proof of this theorem, we note that since the reduced solution is not smooth along certain curves, we must supplement it with some interior layer terms. To simplify our proof, we let $I L(t+a x=b)$ be an interior layer of a solution with the form

$$
W(x, t, \varepsilon)=\left\{\begin{array}{l}
w_{0} \text { for } t+a x \geq b, \\
w_{1} \text { for } t+a x \leq b
\end{array} \text { where } a \text { and } b\right. \text { are constants, then }
$$




$$
\begin{aligned}
I L(t+a x=b)= & O\left(\varepsilon^{1 / 2}\left|\frac{\partial w_{1}}{\partial n}-\frac{\partial w_{0}}{\partial n}\right| \exp \left[\frac{-|t+a x-b|}{\varepsilon^{1 / 2}}\right]\right) \\
& +O\left(\left|w_{1}-w_{0}\right| \exp \left[\frac{-(t+a x-b)^{2}}{\varepsilon^{1 / 2}}\right]\right)+O\left(\varepsilon^{1 / 2}\right),
\end{aligned}
$$

$\partial w / \partial n$ defined by $[\partial \omega / \partial x, \partial \omega / \partial t] \cdot\left[n_{1}, n_{2}\right]$, and $n=\left[n_{1}, n_{2}\right]$ is the outer unit normal to the subdomain $\{(x, t): t+a x-b<0\}$ (cf. [7]).

For the case $f\left(u, u_{x}\right)=h\left(u_{x}\right)$ and $g \neq 0$, the reduced $V$-equation

$$
V_{t}-h\left(U_{x}\right) V_{x}+g\left(x, U, U_{x}\right) V=0
$$

has the solution $V(x, t)=\phi_{2}\left(x-\int_{0}^{t} h(U(x(\tau), \tau)) \mathrm{d} \tau\right)\left[\exp \left(-\int_{0}^{t} g\left(x, U(x, \tau), U_{x}(x, \tau)\right) \mathrm{d} \tau\right\}\right]$ on

$$
B_{<}=\{(x, t) \in B: \sigma(x, t)<0\}=B,
$$

here the parabolic boundary $B=\{(0, t): 0<t<T\} \cup\{(x, 0): 0 \leq x \leq 1\} \cup\{(1, t): 0<t<T\}$ as in Section 2. However, for $(x, t)$ in $B_{0} \cup B_{>}$, where $B_{0}=\{(x, t) \in B: \sigma(x, t)=0\}$ and $B_{>}=\{(x, t) \in B: \sigma(x, t)>0\}$, the solution $V$ cannot satisfy the boundary data. By a similar analysis, we expect a more complicated asymptotic behavior of solutions of system (1.1) as $\varepsilon \rightarrow 0^{+}$in this case. However, there are no explicit expressions for the solutions. A numerical study for $f\left(u, u_{x}\right)=h\left(u_{x}\right)$ and $g \neq 0$ will be given elsewhere.

\section{COMPARISON AND CONCLUSION}

To compare the asymptotic behavior of the time-dependent solution $v$ of (MI) and (MII) with the asymptotic solution $v$ as $\varepsilon \rightarrow 0^{+}$of the steady-state system of (1.1), we summarize the asymptotic behavior of the steady-state (time $t$ tends to infinity) solutions as $\varepsilon \rightarrow 0^{+}$in Figs 5, 6 (cf. [5]). We then briefly summarize the results of the theorem (see Section 4) in Table 1 .

We note that the boundary layer behavior of the time-dependent solution $v$ as $\varepsilon \rightarrow 0^{+}$of (MI):

$$
\begin{aligned}
v(x, t, \varepsilon)= & \phi_{2}\left(x-\int_{0}^{t} h(U(x(\tau), \tau)) \mathrm{d} \tau\right)\left[\exp \left\{-\int_{0}^{t} g\left(x, U(x, \tau), U_{x}(x, \tau)\right) \mathrm{d} \tau\right\}\right] \\
& +B L(x=0)+B L(x=1)
\end{aligned}
$$

is invariant under various boundary data $v$, it is quite different from the steady-state solution showed in Fig. 5. However, we are able to determine the asymptotic behavior of the solutions of (MII) according to the signs of the boundary data $v$, it is the same as its steady-state solution of Model II. Moreover, under certain conditions and for large time, there are similar patterns between the boundary and interior layer behavior of the solution of the time-dependent system (MII) and its steady-state solution of Model II as $\varepsilon \rightarrow 0^{+}$. Nevertheless, the process of the transition from the unsteady-state to the steady-state must be very complicated, even though the steady-state solution of (1.1) is stable with respect to all initial perturbations as $\varepsilon \rightarrow 0^{+}$ (cf. [5]). A numerical study may provide more information. 


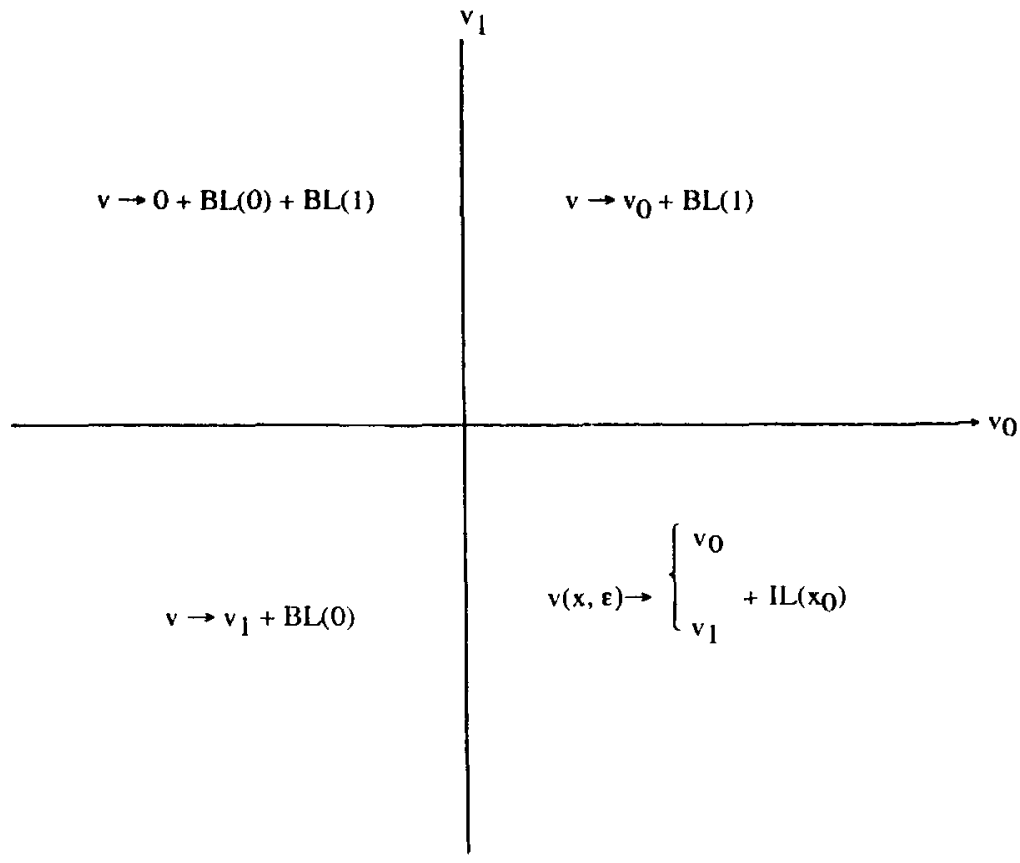

Fig. 5. Boundary value portrait for Model I: $u^{\prime \prime}=v, \varepsilon v^{\prime \prime}+h(u) v^{\prime}=0$.

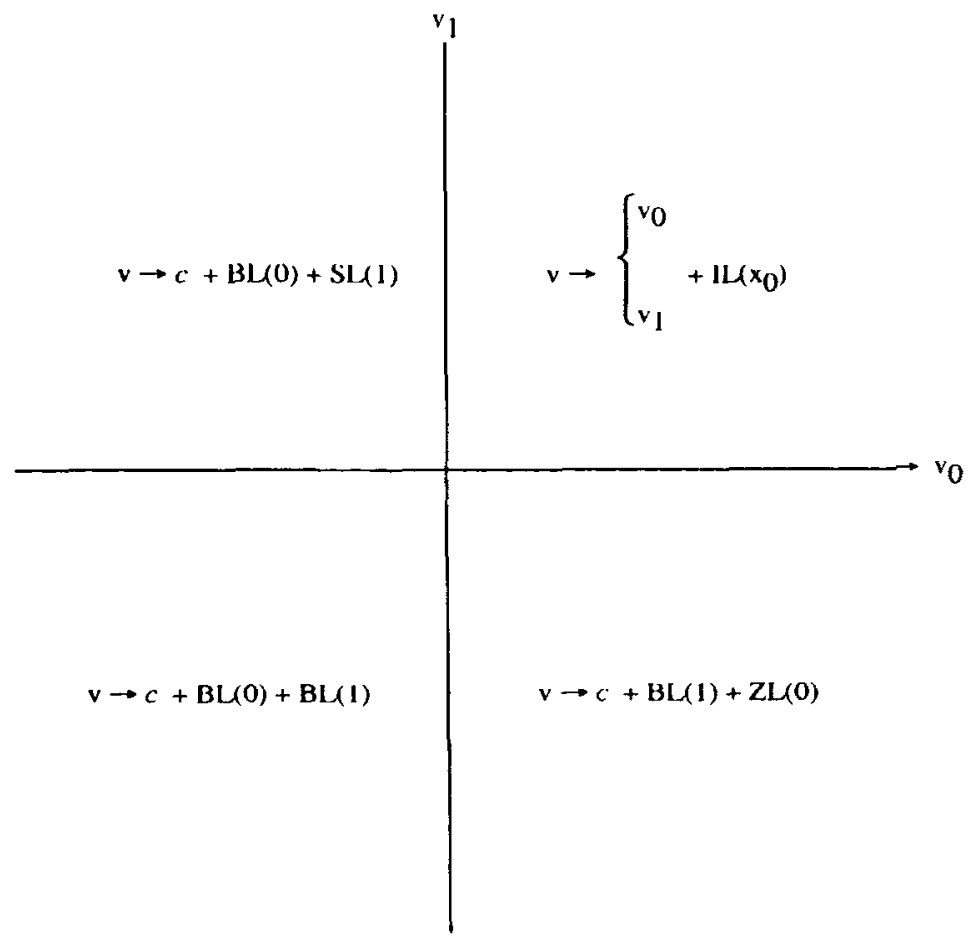

Fig. 6. Boundary value portrait for Model II: $u^{\prime \prime}=v, \varepsilon v^{\prime \prime}+h\left(u^{\prime}\right) v^{\prime}=0$. 
Table 1 .

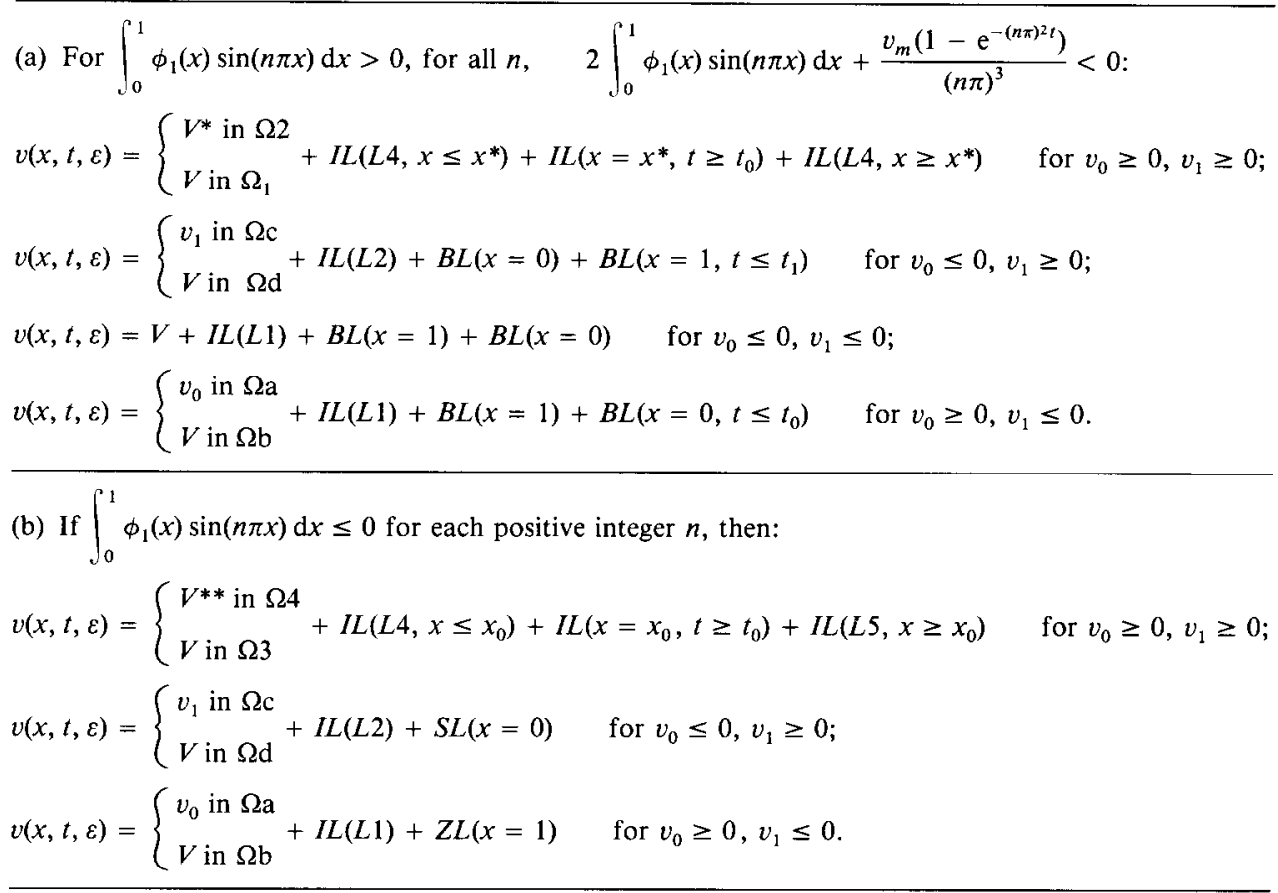

\section{Conclusion}

Singular perturbation theory and asymptotic analysis are used to describe the boundary and interior layer behavior in the solutions of the system as the small positive parameter tends to zero for a finite time. The asymptotic solutions as $\varepsilon \rightarrow 0^{+}$of the $v$-equation of (MI) only depend on the initial data and it is supplemented by the boundary layer at both $x=0$ and $x=1$. The asymptotic solutions as $\varepsilon \rightarrow 0^{+}$of (MII) can be obtained if $\int_{0}^{1} \phi_{1}(x) \sin (n \pi x) \mathrm{d} x$ do not change sign for all positive integers $n$, or we know the signs of

$$
2 \int_{0}^{1} \phi_{1}(x) \sin (n \pi x) \mathrm{d} x+\frac{v_{m}\left(1-\mathrm{e}^{-(n \pi)^{2} t}\right)}{(n \pi)^{3}}
$$

for some $t \geq t_{i}(i=0,1)$ in $[0, T]$ for all $n$. The situations become much more complicated if $\int_{0}^{1} \phi_{1}(x) \sin (n \pi x) \mathrm{d} x$ has oscillations for some $n$.

The reduced solutions $V$ will oscillate if the partial derivative $U_{x}$ oscillates at $x=0$ or $x=1$. The author is studying these situations by using some numerical-asymptotic techniques and the structure of the interior layer of the solutions of (1.1) will also be investigated.

\section{REFERENCES}

1. Dorr F. W. \& Parter S. V., Singular perturbations of nonlinear boundary value problems with turning points, J. math. Analysis Applic. 29, 273-293 (1970).

2. Dorr F. W., Parter S. V. \& Shampine L. F., Applications of the maximum principle to singular perturbation problems, SIAM Rev. 15, 43-88 (1973). 
3. Howes F. A. \& Sh九o S., Asymptotic analysis of model problems for a coupled system, Nonlinear Analysis 13(9), 1013-1024 (1989).

4. HARRIS W. A. JR. \& Shao S., Refined approximations to the solutions of a coupled system with turning points, J. diff. Eqns 9, 125-144 (1991).

5. Shao S., Asymptotic behavior of solutions of model problems for a coupled system, J. math. Analysis Applic. (in press).

6. Rhines P. B. \& Young W. R., How rapidly is a passive scalar mixed with closed streamlines, J. Fluid Mech. 133, $133-145$ (1983).

7. Howes F. A., Multi-dimensional initial-boundary value problems with strong nonlinearities, Archs ration. mech. Analysis 91, 153-168 (1986).

8. CANnON J. R., The one-dimensional heat equations, in Encyclopedia of Mathematics and its Application, Vol. 23. Addison-Wesley, Reading, Massachusetts (1984).

9. Harberman R., Elementary Applied Partial Differential Equations. Prentice-Hall, Englewood Cliffs, New Jersey (1983).

10. Howes F. A., Perturbed boundary value problems whose reduced solutions are nonsmooth, Indiana Univ. math. J. 30, 267-280 (1981). 